\title{
Perfil funcional de saúde física e psicológica do idoso residente na comunidade:
}

\section{Revisão integrativa}

\author{
Functional profile of physical and psychological health of the elderly resident in the community: \\ Integrative review \\ Perfil funcional de salud física y psicológica del mayor residente de la comunidad: Revisión \\ integrativa
}

Recebido: 23/06/2021 | Revisado: 29/06/2021 | Aceito: 01/07/2021 | Publicado: 14/07/2021

\author{
José Rogécio de Sousa Almeida \\ ORCID: https://orcid.org/0000-0003-3722-9354 \\ Faculdade do Vale do Jaguaribe, Brasil \\ E-mail: rogeciofisio@gmail.com \\ Micássio Andrade \\ ORCID: https://orcid.org/0000-0003-2836-9080 \\ Universidade do Estado do Rio Grande do Norte, Brasil \\ E-mail: micassiofernandes@gmail.com \\ Jean Michel Regis Mendes \\ ORCID: https://orcid.org/0000-0001-5353-5963 \\ Universidade do Estado do Rio Grande do Norte, Brasil \\ E-mail: jeanmendesfisio@gmail.com \\ Heloísa Alencar Duarte \\ ORCID: https://orcid.org/0000-0003-0980-0277 \\ Faculdade de Enfermagem Nova Esperança de Mossoró, Brasil \\ E-mail: heloisaalencar4@gmail.com \\ Elihab Gomes Pereira \\ ORCID: https://orcid.org/0000-0002-2461-4096 \\ Universidade do Estado do Rio Grande do Norte, Brasil \\ E-mail: elihabpsi@gmail.com \\ Lucidio Clebeson de Oliveira \\ ORCID: https://orcid.org/0000-0002-2033-7546 \\ Universidade do Estado do Rio Grande do Norte, Brasil \\ E-mail: lucidioclebeson@uern.br
}

\begin{abstract}
Resumo
Objetivou-se identificar o perfil de funcionalidade da pessoa idosa brasileira residente na comunidade acompanhada pelas equipes de saúde da atenção primária. Tratou-se de revisão integrativa desenvolvida entre março e julho de 2020, utilizando-se a estratégia de busca "Avaliação geriátrica” AND "Atenção Primária à Saúde”; "Geriatric Assessment" AND "Primary Health Care”. Obteve-se um achado inicial de 8.146 artigos que, após aplicação dos filtros e dos critérios de elegibilidade, cinco artigos foram incluídos para a análise final, considerando os aspectos físicos e psicológicos da pessoa idosa. Observou-se que fatores sociodemográficos influenciam diretamente na dependência do idoso. Sintomas depressivos, número de comorbidades, prática atividade física, auto avaliação da sua saúde e amparo familiar são influenciadores para fragilidade e dependência desencadeando baixa funcionalidade física e psicológica da pessoa idosa comprometendo sua autonomia para desempenhar funções do dia a dia. Concluiu-se que a funcionalidade do idoso é diretamente influenciada por fatores extrínsecos, sendo necessário um redirecionamento das práticas profissionais para a efetivação de uma atenção integral à saúde.
\end{abstract}

Palavras-chave: Geriatria; Atendimento primário à saúde; Classificação internacional de funcionalidade; Incapacidade e saúde.

\begin{abstract}
The objective was to identify the functionality profile of the Brazilian elderly living in the community accompanied by primary care health teams. This was an integrative review developed between March and July 2020, using the search strategy "Geriatric Assessment" AND "Primary Health Care", and "Geriatric Assessment" AND "Primary Health Care". An initial finding of 8,146 articles was obtained. After applying the filters and eligibility criteria, five articles were included for the final analysis, considering the physical and psychological aspects of the elderly person. It was observed that sociodemographic factors directly influence the dependence of the elderly. Depressive symptoms, number of comorbidities, physical activity, self-assessment of their health and family support influence frailty and dependence, triggering low physical and psychological functionality in the elderly, compromising their autonomy to
\end{abstract}


perform daily functions. It was concluded that the functionality of the elderly is directly influenced by extrinsic factors, requiring a redirection of professional practices for the realization of comprehensive health care.

Keywords: Geriatrics; Primary health care; International classification of functionality; Disability and health.

\section{Resumen}

El objetivo fue identificar el perfil de funcionalidad de los ancianos brasileños residentes en la comunidad acompañados de equipos de salud de atención primaria. Revisión integradora desarrollada entre marzo y julio de 2020, utilizando la estrategia de búsqueda "Evaluación geriátrica" Y "Atención primaria de salud", y "Evaluación geriátrica" Y "Atención primaria de salud". Se obtuvo un hallazgo inicial de 8.146 artículos, luego de aplicar los filtros y criterios de elegibilidad, se incluyeron cinco artículos para el análisis final, considerando los aspectos físicos y psicológicos del anciano. Se observó que factores sociodemográficos influyen directamente en la dependencia de los adultos mayores. Los síntomas depresivos, el número de comorbilidades, la actividad física, la autoevaluación de su salud y el apoyo familiar influyen en la fragilidad y la dependencia, desencadenando una baja funcionalidad física y psicológica en los ancianos, comprometiendo su autonomía para realizar las funciones diarias. Se concluyó que la funcionalidad del anciano está directamente influenciada por factores extrínsecos, requiriendo una reorientación de las prácticas profesionales para la realización de una atención integral de salud.

Palabras clave: Geriatría; Primeros auxilios; Clasificación internacional de funcionalidad; Discapacidad y salud.

\section{Introdução}

O número crescente da população idosa brasileira (Pamplona et al, 2020) e em escala global tem despertado relevante interesse nos fatores que influenciam a qualidade de vida e a funcionalidade da pessoa idosa (Costanzi et al, 2018). Com o passar dos anos, há alterações naturais do envelhecimento a nível fisiológico que refletem na capacidade da pessoa idosa de se manter ativa e autônoma (Sousa et al, 2012). Porém, há fatores externos como estilo de vida, uso de álcool, fumo e patologias que podem acelerar o processo de dependência do idoso (Karen et al, 2015), já que o envelhecimento acarreta mudanças significativas nos aspectos físicos, psicológicos e sociais (Camargos et al, 2015), preservando ou não sua capacidade funcional em cada aspecto.

Compreende-se como capacidade funcional do idoso, sua habilidade de realizar tarefas físicas, possuindo preservadas suas atividades mentais, além de manter relações sociais adequadas (Matsudo et al, 2002). As mudanças negativas na capacidade funcional do idoso geram grande impacto na sua saúde, nas relações familiares, sociais e na assistência profissional, podendo necessitar de ajuda multiprofissional, o que muitas vezes não é possível a nível da atenção primária à saúde (APS).

Dessa forma, é importante que os profissionais da assistência primária conheçam os determinantes em saúde que contribuem para a independência funcional da pessoa idosa para que possam avaliar o idoso em todos os seus aspectos sendo capazes de ampliar a atenção à saúde, como sugerido pela Política Nacional de Saúde da Pessoa Idosa (Brasil, 2006), contribuindo para o retardo e prevenção das incapacidades (Matsudo et al, 2002), oriundas do envelhecimento. Nesse sentido, objetivou-se identificar o perfil de funcionalidade da pessoa idosa brasileira residente na comunidade acompanhada pelas equipes de saúde da atenção primária.

\section{Metodologia}

Este estudo se caracteriza como uma revisão integrativa (Botelho, Cunha e Macedo, 2011) desenvolvida a partir da seguinte pergunta norteadora: "Qual o perfil de funcionalidade da pessoa idosa brasileira residente na comunidade atendida pela atenção primária à saúde?” O processo de seleção e buscas de estudos nas bases de dados aconteceu no período de março a julho de 2020, e foi realizado nas seguintes bases: Lilacs, PubMed, Scielo, Scopus e Web of Science.

Utilizou-se como critérios de inclusão os estudos que atendessem aos seguintes pré-requisitos: a) dados primários; b) publicados entre 2016 a 2020; c) disponíveis como textos na íntegra e d) escritos em língua inglesa ou portuguesa. Descartou- 
se os estudos que: a) não estiverem disponíveis de forma gratuita; b) não tivesse relação direta com a temática pesquisada e c) não fossem realizados no Brasil.

Os descritores selecionados foram “Avaliação geriátrica” e "Atenção Primária à Saúde”, os quais foram utilizados nas bases Lilacs e Scielo e "Geriatric Assessment” e "Primary Health Care” usados nas bases PubMed, Scopus e Web of Science, realizando a interação entre os descritores através do operador booleano AND: “Avaliação geriátrica” AND “Atenção Primária à Saúde”; “Geriatric Assessment” AND "Primary Health Care”.

No processo de busca teve-se um achado inicial de 8.146 estudos. A partir do quantitativo final após uso dos filtros, os estudos encontrados foram exportados para o Endnote ${ }^{\mathrm{TM}}$ desktop versão 20 da empresa Clarivate ${ }^{\mathrm{TM}}$. Neste software realizou-se a eliminação de estudos duplicados, reduzindo de 729 para 575 artigos. Após a seleção por título restaram 19 estudos. Agrupou-se os estudos por aspectos: físicos, psicológicos e cognitivos, finalizando com um total final de cinco produções, sendo três do eixo psicológico e dois do eixo físico, não sendo selecionada nenhuma do eixo cognitivo.

A seleção dos textos completos e de suas informações foi realizada a partir da ficha URSi (Ursi 2005) e do fluxograma do processo de seleção dos textos com a utilização do Checklist PRISMA (Moher et al, 2009), como observado na Figura 1.

Figura 1. Busca e seleção dos estudos incluídos nesta revisão.
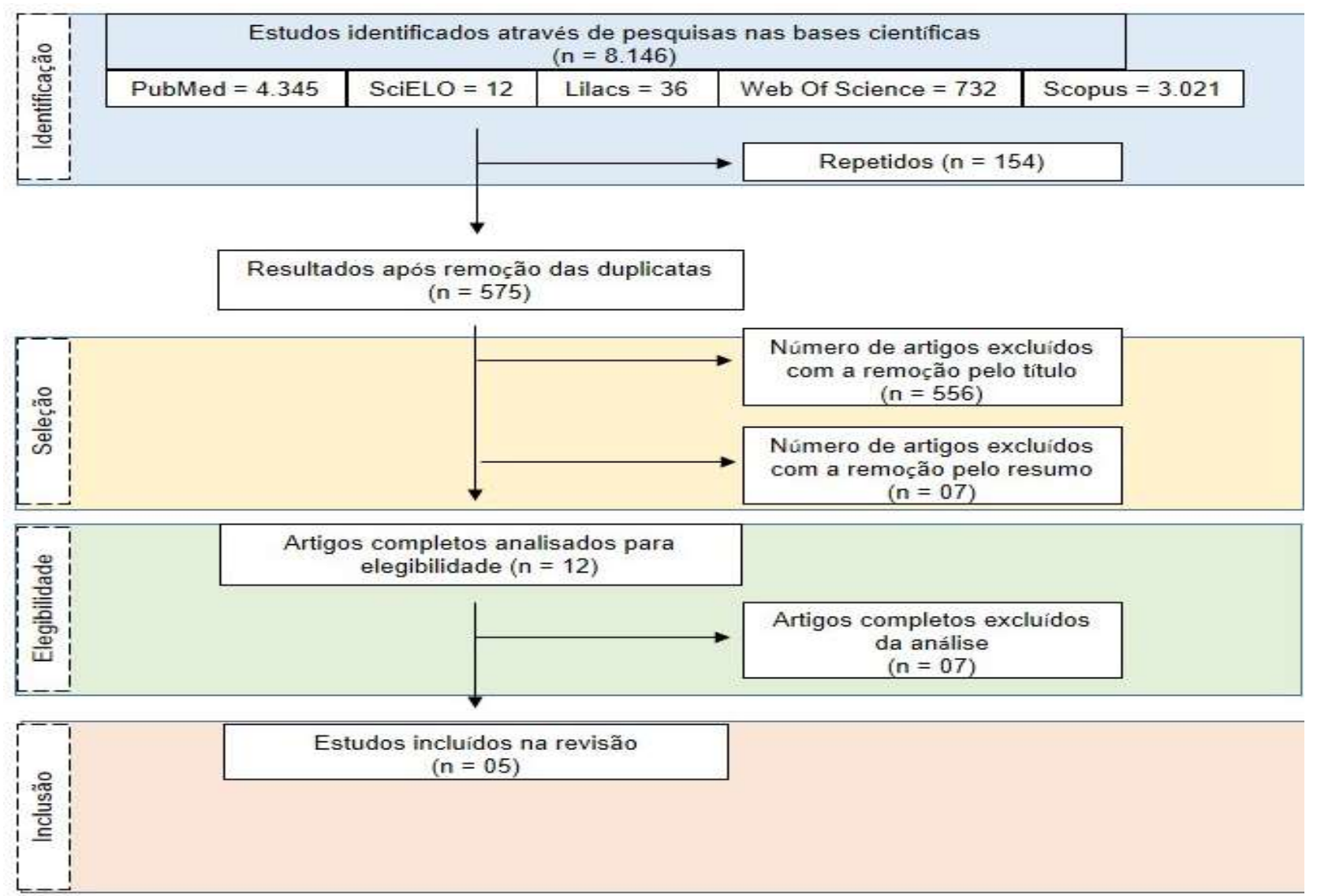


\section{Resultados e Discussão}

O condensado dos estudos selecionados para análise final foram organizados a partir dos autores, ano de publicação, tipo de estudo, instrumentos utilizados na coleta das informações e os principais resultados. Os estudos foram publicados entre 2016 e 2020, todos do tipo transversal, como observa-se no quadro abaixo.

Quadro 1. Descrição dos estudos selecionados.

\begin{tabular}{|c|c|c|c|c|}
\hline Autores & Ano & Tipo de estudo & Instrumentos utilizados & Resultados \\
\hline Cordeiro RC. et al. & 2020 & Transversal & $\begin{array}{l}\text { Brazil Old Age Shedule; Mini } \\
\text { Exame do Estado Mental; } \\
\text { Escala de Depressão Geriátrica; } \\
\text { Escala de Resiliência; Escala de } \\
\text { Apoio Social; Inventário de } \\
\text { Eventos Estressantes; Escala de } \\
\text { Satisfação com a Vida e a } \\
\text { Escala de Afetos Positivos e } \\
\text { Negativos. }\end{array}$ & $\begin{array}{l}\text { Há associação entre as } \\
\text { variáveis } \\
\text { sociodemográficas e } \\
\text { sintomas depressivos, } \\
\text { estado mental, } \\
\text { resiliência e apoio } \\
\text { social. }\end{array}$ \\
\hline Gato JM, et al. & 2018 & Transversal & $\begin{array}{l}\text { Whoqol-old/Whoqol-bref; } \\
\text { Escala de Depressão Geriátrica; } \\
\text { Questionário sociodemográfico } \\
\text { e clínico. }\end{array}$ & $\begin{array}{l}\text { Houve associação entre } \\
\text { sintomas depressivos e } \\
\text { diminuição da QV em } \\
\text { todos os domínios e a } \\
\text { satisfação com a QV foi } \\
\text { moderada. }\end{array}$ \\
\hline Lenardt MH, et al. & 2016 & Transversal & \begin{tabular}{llr} 
Revisão & do & \multicolumn{2}{c}{ prontuário } \\
observando & o diagnóstico de \\
depressão da Classificação & da Ças. \\
Internacional de Doençana
\end{tabular} & $\begin{array}{l}\text { Os níveis de tratamento } \\
\text { da depressão em idosos } \\
\text { atendidos no PSF são } \\
\text { muito baixos. }\end{array}$ \\
\hline $\begin{array}{l}\text { Brito KQD, Menezes } \\
\text { TN, Olinda RA. }\end{array}$ & 2016 & Transversal & IMC; Índice de Barthel; & $\begin{array}{l}\text { Há associação entre } \\
\text { incapacidade funcional, } \\
\text { número de doenças } \\
\text { crônicas, condições de } \\
\text { saúde auto avaliadas e } \\
\text { prática de atividades } \\
\text { físicas. }\end{array}$ \\
\hline Bárbara FA, et al. & 2017 & Transversal & $\begin{array}{l}\text { Minnesota Leisure Activity } \\
\text { Questionnaire; } \quad \text { Escala de }\end{array}$ & $\begin{array}{l}\text { Há associação entre } \\
\text { capacidade funcional e }\end{array}$ \\
\hline
\end{tabular}




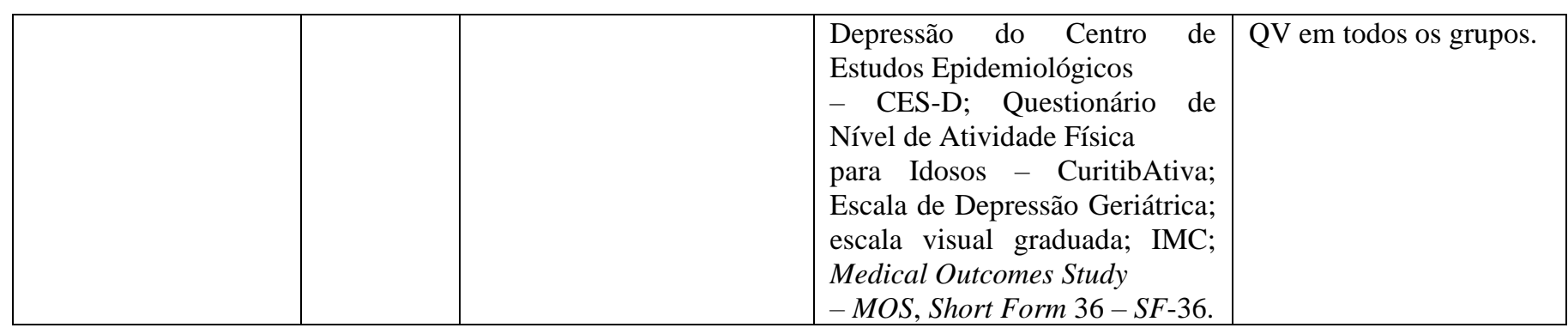

Fonte: Autores (2020).

Observou-se uma variedade de instrumentos aplicados para avaliar a funcionalidade da pessoa idosa tanto no rastreio da depressão e demência (Veras et al, 2020; Krieger et al, 2020) quanto nas avaliações físicas (Araujo et al, 2020; Silva et al, 2020). Nenhum dos estudos utilizou a Classificação Internacional de Funcionalidade, Incapacidade e Saúde (CIF) (OMS, 2013). No que diz respeito ao perfil do eixo psicológico, viu-se que há uma relação entre as condições de vida e o surgimento de sintomas depressivos que podem afetar o estado mental e a forma como o idoso reage a esses desajustes, tendo impactos diretos na qualidade de vida. No que tange à capacidade funcional física, os idosos que apresentam doenças crônicas e baixa prática de atividade física se apresentaram como mais propensos a incapacidade funcional afetando negativamente sua qualidade de vida.

A capacidade funcional se apresenta como uma condição multifatorial, podendo ser influenciada por fatores sociodemográficos e epidemiológicos (Cordeiro et al, 2020), o que se observou em dois estudos analisados que evidenciaram haver associação entre as variáveis sociodemográficas como saúde física, utilização de serviços médicos, dentários e saúde mental com sintomas depressivos (Gato et al, 2016) e depressão (Lenardt et al, 2016), percebendo-se uma relação entre sintomas depressivos e satisfação moderada da qualidade de vida, assim como também associação entre capacidade funcional física e qualidade de vida (Brito et al, 2016).

Observou-se que a incapacidade funcional para as atividades instrumentais de vida diária (AIVD) somada a percepção negativa de saúde, polifarmácia e número elevado de morbidades são fatores associados e desencadeadores de pré-fragilidade e fragilidade em pessoas idosas (Cordeiro et al, 2020), o que se assemelha aos resultados encontrados, mostrando que quanto maior o número de doenças crônicas, menor foi a avaliação de saúde contribuindo para uma significativa incapacidade funcional (Bárbara et al, 2017).

Pessoas idosas com maior idade, maior número de morbidades e com presença indicativa de depressão estão mais predispostas a incapacidade funcional para as atividades básicas de vida diária, já menor escolaridade e renda baixa são fatores para incapacidade funcional relacionado as atividades instrumentais de vida diária (Abrantes et al, 2019).

A autopercepção de saúde dos idosos parece não ser fiel ao seu real estado de saúde. Uma pesquisa realizada com idosos e seus cuidadores mostrou que os idosos possuem autopercepção positiva da sua qualidade de vida, divergindo da opinião dos seus cuidadores. No mesmo sentido, idosos se consideram saudáveis embora apresentassem muitos agravos à saúde, inclusive quadros de depressão (Abrantes et al, 2019). Um dos estudos da nossa análise se aproxima dos achados anteriores ao evidenciar que a maioria dos idosos avaliados consideraram seu estado de saúde psicológico saudável mesmo fazendo uso de ansiolíticos e tendo moderada satisfação em relação a sua qualidade de vida (Lenardt et al, 2016).

Cordeiro e colaboradores (2020) também observaram associação entre as variáveis sociodemográficas e sintomas depressivos, estado mental, resiliência e apoio social, sendo as mulheres mais satisfeitas com a vida, apresentando mais afetos positivos e menos afetos negativos do que os homens (Gato et al, 2016). Ainda, evidenciaram um perfil de idosos que não apresentaram declínio de saúde mental, que vivenciam frequentemente os afetos positivos e estão satisfeitos com a vida (Gato et al, 2016), o que se assemelha aos achados de um estudo realizado na atenção básica de saúde de João Pessoa - PB 
observando que os idosos estavam satisfeitos com a sua vida e que se sentiam felizes, figurando como fator de proteção contra os sintomas depressivos (ChiloffI et al, 2018).

Já entre os idosos paulistanos, a prevalência de sintomas depressivos foi de 14,2\% em 2006 associando-se a eles piores condições de saúde, dependência para as atividades cotidianas, disfunção familiar, maior número de doenças, percepção negativa de sua saúde e memória (Scazufca et al, 2016). Nota-se que os níveis de tratamento da depressão em idosos atendidos no programa saúde da família (PSF) são muito baixos em comparação com as taxas de tratamento de diabetes e hipertensão, por exemplo, sendo necessário melhorar o tratamento da depressão no PSF a fim de melhorar a qualidade dos cuidados da pessoa idosa (Gonçalves et al, 2013).

No entanto, profissionais enfermeiros da ESF afirmaram não possuir formação específica para lidar com questões de saúde mental, sentindo-se despreparados para lidar com as demandas do serviço, necessitando de aperfeiçoamento na área com propostas de educação permanente (Coelho et al, 2009). Torna-se necessário que diferentes profissionais passem a incluir idosos em atividades com valor social e ofereçam apoio psicossocial a suas famílias para minimizar complicações clínicas, expandir a QV e fortalecê-los para um maior nível possível de autonomia (Lenardt et al, 2016).

Nesse sentido, destaca-se a atenção básica à saúde como um espaço importante do cuidar e, portanto, sendo capaz de realizar o rastreio e o monitoramento de sintomas depressivos em idosos (ChiloffI et al, 2018; Santos et al, 2020) e desenvolver ações de prevenção a baixa funcionalidade física para retardar o surgimento das incapacidades (Gonçalves et al, 2013).

\section{Conclusão}

Observou-se que o perfil de funcionalidade da pessoa idosa brasileira residente na comunidade está associado a fatores sociodemográficos que influenciam diretamente a sua dependência ou independência. Percebeu-se que o idoso com sintomas depressivos, com elevado número de comorbidades, que não pratica atividade física, com baixa avaliação de sua saúde e que não se sente amparado por familiares tendem a se tornar mais frágeis e mais dependentes, com baixa funcionalidade física e psicológica.

Destaca-se que a avaliação funcional da pessoa idosa residente na comunidade não é comumente realizada de forma global pelos profissionais desse nível de atenção à saúde, necessitando de reflexão das práticas profissionais. Esta pesquisa limitou-se a uma análise reduzida de estudos encontrados relacionados a funcionalidade física e psicológica do idoso residente na comunidade. Assim, sugere-se que mais estudos como este sejam realizados bem como pesquisas no âmbito da saúde do idoso na atenção primária a fim de ampliar os conhecimentos já existentes e fomentar a prática profissional melhorando a vida das pessoas idosas.

\section{Referências}

Abrantes, G. G., Souza, G.G., Cunha, N. M., Rocha, H. N. B., Silva, A. O., \& Vasconcelos, S. C. (2019). Sintomas depressivos em idosos na atenção básica à saúde. Rev. Bras. Geriatr. Gerontol, 22 (4). https://doi.org/10.1590/1981-22562019022.190023.

Araujo, E. A. T., de Lima Filho, B. F., da Silva, A. C. M. B., de Melo, M. C. S., Gazzola, J. M., \& da Costa Cavalcanti, F. A. (2020). A utilização do Índice de Barthel em idosos brasileiros: uma revisão de literatura. Revista Kairós: Gerontologia, 23(2), 217-231.

Bárbara, F. A., Dias, F. A., Ferreira, P. C. S., Gomes, N. C., \& Tavares, D. M. S. (2017) Incapacidade funcional e morbidades entre idosos, segundo condições sociodemográficas e indicativo de depressão. Invest. educ. enferm, 35(1). Disponível em: https://doi.org/10.17533/udea.iee.v35n1a06.

Botelho, L. L. R., Cunha, C. C. A., \& Macedo, M. (2011). O método da revisão integrativa nos estudos organizacionais. Gestão e Sociedade. Belo Horizonte, $5(2), 11,121-136$.

Brasil. (2006). Política Nacional de Saúde da Pessoa Idosa. Portaria nº 2.528, de 19 de outubro de 2006.

Brito, K. Q. D., Menezes, T. N., Olinda, R.A. (2016). Incapacidade funcional: condições de saúde e prática de atividade física em idosos. Rev Bras Enferm, 69 (5): $825-32$. 
Camargos, M. C. S., Marcos, R. G. (2015). Viver mais e melhor? Estimativas de expectativa de vida saudável para a população brasileira. Caderno de Saúde Pública, 31 (7): 1460-1472.

ChiloffI, C. L. M., Lima, M. C. P., Torres, A.R., Santos, J. L. F., Duarte, Y. D., Lebrão, M. L., et al. (2018). Sintomas depressivos em idosos do município de São Paulo, Brasil: prevalência e fatores associados (Estudo SABE). Rev bras epidemiol, 21(2).

Coelho, C. F., Burini, R. C. (2009). Atividade física para prevenção e tratamento das doenças crônicas não transmissíveis e da incapacidade funcional. Revista de Nutrição, 22 (6): 937-946.

Cordeiro, R. C., Santos, R. C., Araújo, G. K. N., Nascimento, N. M., Albanita, R. Q. S. (2020). Perfil de saúde mental de idosos comunitários: um estudo transversal. Rev Bras Enferm. 73 (1). Disponível em: https://doi.org/10.1590/0034-7167-2018-0191.

Costanzi, R. N., Fernandes, A. Z., Santos, C. F. D., \& Sidone, O. J. G. (2018). Breve análise da nova projeção da população do IBGE e seus impactos previdenciários.

Gato, J. M., Zenevicz, L. T., Madureira, V. S. F., Silva, T. G., Celich, K. L. S., Souza, S. S. (2018). Saúde mental e qualidade de vida de pessoas idosas. Av Enferm, 36 (3): 302-310. Disponível em: doi: https://doi.org/10.15446/av.enferm.v36n3.68498.

Gonçalves, R., Pedrosa, L., Oliveira, M., Silva, Q., Abreu, R., Pinho, P. (2013). Promoção da saúde mental: ações dos enfermeiros inseridos na atenção primária. Revista Portuguesa de Enfermagem de Saúde Mental, 10 (49).

Karen, B. R., Seplaki, C. L., Huang, J., Buta, B., Kalyani, R. R., Varadhan, R. (2015). Frailty in older adults: a nationally representative profile in the United States. J. Gerontol A Biol Sci Med Sci, 70 (11): 1427-1434.

Krieger, D. M., Coronel, L. C. I., Lima L. D. (2020). The relevance of Mini Mental State Examination (MMSE) use on demential interdiction exams in judicial proceedings. J. bras. Psiquiatr, 69(1): 73-77.

Lenardt, M. H., Carneiro, N. H. K., Binotto, M. A., Willig, M. H., Lourenço, T. M., Albino, J. (2016). Fragilidade e qualidade de vida de idosos usuários da atenção básica de saúde. Rev Bras Enferm, 69 (3): 478-83.

Matsudo, V. K., Matsudo, S. M., Andrade, D. R., Araújo, T. L., Oliveira, L. C., Bragion, G. F. (2002). Promotion of physical activity in a developing country: The Agita São Paulo experience. Public Health Nutr, 5 (1a): 1-10.

Melo, B. L., Kenya, N., Santos, C. M. C., \& Jorge, M. C. (2020). Perfis de integração social entre idosos institucionalizados não frágeis no município de Natal, Rio Grande do Norte, Brasil. Ciênc. Saúde coletiva, 25 (6): 2017-2030.

Moher, D., Liberati, A., Tetzlaff, J., Altman, D. G., Altman, D., \& Antes, G, et al. (2009). Preferred reporting items for systematic reviews and meta-analyses: The PRISMA statement. PLoS Med; 6 (7)

Organização Mundial da Saúde. (2013). Como usar a CIF: Um manual prático para o uso da Classificação Internacional de Funcionalidade, Incapacidade e Saúde (CIF). OMS.

Pamplona, D. A., Pinheiro, D. M., Fachin, M. G., \& Passos, R. M. (2020). Novas reflexões sobre o pacto global e as ODS da ONU. E-book (PDF): ISBN 97865-87027-00-5. NCA Comunicação e Editora.

Santos, M. A. B., Silveira, M. R., Fernanda, F. P., Carneiro, G. G., \& Lima, S. V. (2020). Fatores associados à violência contra o idoso: uma revisão sistemática da literatura. Ciênc. Saúde coletiva, 25(6): 2153-2175.

Scazufca, M., Menezes P., Tabb K., Kester, R., Wulf, R. W., \& Hsiang, H. H. (2016). Identification and treatment of depression of older adults in primary care: findings from the São Paulo Ageing and Health Study. Family Practice, 33 (3): $233-237$.

Silva, F. G., Oliveira, C. B., Hisamatsu, T. M., Negrão Filho, R. F., Rodrigues, C. R. D., \& Franco, M. R., et al. (2020). Critical evaluation of physical activity questionnaires translated To Brazilian-Portuguese: a systematic review on cross-cultural adaptation and measurements properties. Brazilian journal of physical therapy, 24(3), 187-218.

Sousa, A. C. P. A., Dias, R. C., Maciel, A. C. C., \& Guerra, R. O. (2012). Frailty syndrome and associated factors in community-dwelling elderly in Northeast Brazil. Arch Gerontol Geriatrics, 54 (2): 95-101.

Trentini, C. M., Chachamovich, E., Figueiredo, M., Hirakata, V. N., \& Fleck, M. P. A. (2006). A percepção de qualidade de vida do idoso avaliada por si próprio e pelo cuidador. Estudos de Psicologia, 11(2), 191-197.

Ursi, E. S. Prevenção de lesões de pele no perioperatório: revisão integrativa da literatura. (2005). 130 f. Dissertação (Mestrado em Enfermagem) - Escola de Enfermagem, Universidade de São Paulo, Ribeirão Preto.

Veras, C., Hartle, L., Araujo, V. C., \& Charchat-Fichman, H. (2020). Estudo normativo da Escala de Depressão Geriátrica em amostra de idosos do Rio de janeiro. Neuropsicologia Latinoamericana, 12(3). 